\title{
Preditores de não aderência ao tratamento na psicoterapia psicanalítica de crianças
}

\author{
Predictors of treatment non-adherence in child psychoanalytic psychotherapy \\ Marina Bento Gastaud ${ }^{1}$, Fernando Basso ${ }^{2}$, Juliana Prytula Greco Soares ${ }^{3}$, Claudio Laks Eizirik ${ }^{4}$, Maria Lucia
Tiellet Nunes $^{5}$
}

${ }^{1}$ Psicóloga. Doutoranda em Ciências Médicas: Psiquiatria, Universidade Federal do Rio Grande do Sul (UFRGS), Porto Alegre, RS. ${ }^{2}$ Psicólogo. Membro provisório, Centro de Estudos Psicanalíticos de Porto Alegre, Porto Alegre, RS. ${ }^{3}$ Acadêmica de Psicologia, PUCRS, e de Estatística, UFRGS. ${ }^{4}$ Psicanalista. Doutor em Medicina. Professor associado, UFRGS. ${ }^{5}$ Psicóloga. Doutora em Psicologia, Freie Universität Berlin, Berlim, Alemanha. Professora titular, PUCRS.

Estudo realizado no Programa de Pós-Graduação em Psicologia da Pontifícia Universidade Católica do Rio Grande do Sul (PUCRS), Porto Alegre, RS, e no Programa de Pós-Graduação em Psiquiatria da Universidade Federal do Rio Grande do Sul (UFRGS), Porto Alegre, RS.

Suporte financeiro: Conselho Nacional de Desenvolvimento Científico e Tecnológico (CNPq).

\section{Resumo}

Introdução: A alta prevalência de interrupções nas fases iniciais do atendimento psicoterapêutico tem sido demonstrada em estudos nacionais e internacionais. Estudos com pacientes adultos têm demonstrado variáveis sociodemográficas e clínicas associadas à aderência/não aderência à psicoterapia, porém a literatura voltada a crianças é escassa.

Objetivo: Examinar a associação entre variáveis sociodemográficas/clínicas e aderência/não aderência à psicoterapia psicanalítica de crianças.

Método: Trata-se de estudo documental, retrospectivo, realizado a partir dos prontuários de todas as crianças atendidas em duas instituições de atendimento psicológico em Porto Alegre entre 1979 e 2007.

Resultados: Foram analisados 2.106 prontuários, sendo que 1.083 compuseram a amostra final da presente investigação. Destas, $21,5 \%$ não aderiram ao tratamento. A variável fonte de encaminhamento mostrou-se associada ao desfecho, demonstrando que o encaminhamento à psicoterapia por psiquiatras é um fator protetor à não aderência ao tratamento, enquanto que o encaminhamento pela família é um fator de risco para a não aderência.

Conclusão: Conhecer o perfil das crianças que não aderem à psicoterapia possibilita aos terapeutas o estabelecimento de técnicas de intervenção em fases iniciais do tratamento, a fim de facilitar a adesão da família à psicoterapia da criança. Abandono e não aderência à psicoterapia devem ser entendidos por clínicos e pesquisadores como fenômenos distintos, tendo em vista que apresentam preditores diferentes.

Descritores: Psicoterapia, criança, pacientes desistentes do tratamento, recusa do paciente ao tratamento, suspensão de tratamento.

\begin{abstract}
Introduction: The high prevalence of interruptions in the early stages of psychotherapeutic treatment has been shown in national and international studies. Studies conducted with adults have demonstrated association between social, demographic, and clinical characteristics and adherence/nonadherence to psychotherapy; however, literature focused on children is scarce.

Objective: To assess associations between social, demographic, and clinical variables and adherence/non-adherence to treatment in child psychoanalytic psychotherapy.

Method: This is a retrospective study based on the analysis of medical records of all children who received treatment at two psychological clinics in Porto Alegre, southern Brazil, between 1979 and 2007.

Results: A total of 2,106 records were analyzed, and 1,083 children were included in the final sample. Of these, $21.5 \%$ did not adhere to treatment. The variable referral source was associated with the outcome, i.e., referral to psychotherapy by psychiatrists was a protective factor to non-adherence to treatment, whereas referral by the family was a risk factor for non-adherence.

Conclusion: Knowing the profile of children who do not adhere to psychotherapy enables therapists to establish intervention techniques in the early stages of treatment, so as to facilitate family adherence to child psychotherapy. Because dropout and non-adherence to therapy have different predictive factors, they should be considered as distinct phenomena by clinicians and investigators.
\end{abstract}

Keywords: Psychotherapy, child, patient dropout, treatment refusal, withholding treatment.

Correspondência:

Marina Bento Gastaud, Rua Comendador Caminha, 312/205, Bairro Moinhos de Vento, CEP 90430-030, Porto Alegre, RS, Brasil. Tel.: (51) 3346.1664. E-mail: marinagastaud@hotmail.com

Não foram declarados conflitos de interesse associados à publicação deste artigo.

Copyright $($ C Revista de Psiquiatria do Rio Grande do Sul - APRS

Recebido em 06/06/2010. Aceito em 04/07/2010. 


\section{Introdução}

Recentes estudos do Lancet Global Mental Health Group $^{1}$ demonstram que $30 \%$ da população mundial apresenta alguma forma de transtorno mental, o que afeta a funcionalidade do indivíduo e sua qualidade de vida. De acordo com Fleitlich-Bilyk \& Goodman ${ }^{2}, 10$ a 20\% das crianças brasileiras sofrem de doenças psiquiátricas. Existe uma preocupação crescente dos profissionais da área da saúde com a defasagem evidente percebida entre a referida necessidade e a busca por tratamento especializado. Ponderando que essa defasagem fica ainda maior se for considerado o número de crianças que, depois de procurar ajuda, abandona ou não chega a iniciar o tratamento, há a necessidade de que mais estudos investiguem medidas eficientes de manter as crianças que necessitam de psicoterapia em atendimento.

No início do tratamento, ao receber o paciente, o terapeuta deve fazer uma avaliação inicial e decidir pela melhor forma de ajudar cada caso. Tais avaliações preliminares têm o objetivo de verificar indicações ou contraindicações para a demanda, ao mesmo tempo em que se inicia a construção de uma aliança terapêutica entre o paciente e o terapeuta. Após a definição dos objetivos do tratamento e da compreensão do paciente sobre como irá funcionar a psicoterapia, o contrato é estabelecido, e é iniciado o tratamento psicanalítico ${ }^{3}$.

É alta a prevalência de desistências durante esse perío$\mathrm{do}^{4-6}$, e a maior parte da literatura sobre o tema se dedica à população adulta. Investigação metanalítica ${ }^{7}$ sobre interrupção da psicoterapia revisou 125 estudos e encontrou que somente 16 envolviam amostras compostas por crianças. Desde essa revisão, o número de estudos realizados em centros comunitários de saúde mental com crianças continua escasso $^{8}$. No entanto, entender o perfil da clientela que não adere à psicoterapia psicanalítica seria um primeiro passo na elaboração de medidas para tentar engajar essas crianças e seus responsáveis em psicoterapia; também auxiliaria na revisão de critérios de indicação e contraindicação para essa modalidade terapêutica, a fim de atualizar as ferramentas técnicas para a demanda atual de crianças em psicoterapia.

Os objetivos do presente estudo foram: 1) examinar a relação entre variáveis sociodemográficas (idade, sexo, configuração familiar, escolaridade e cidade em que reside) e aderência/não aderência à psicoterapia psicanalítica de crianças; e 2) examinar a relação entre variáveis clínicas (motivo da consulta, fonte de encaminhamento, época do ano em que buscou atendimento e uso de medicação psiquiátrica ou neurológica) e aderência/não aderência à psicoterapia psicanalítica de crianças.

\section{Método}

Foi realizado estudo quantitativo, retrospectivo e descritivo a partir de documentos (materiais sobre atendimento psicanalítico de crianças) arquivados em duas instituições: Contemporâneo - Instituto de Psicanálise e Transdisciplinaridade e Centro de Estudos, Atendimento e Pesquisa da Infância e Adolescência (CEAPIA).

Para inclusão na amostra, adotou-se como critério de faixa etária o estabelecido no Estatuto da Criança e do Adolescente ${ }^{9}$, que considera como criança "a pessoa com até 12 anos incompletos". Todos os prontuários de crianças que buscaram psicoterapia no Contemporâneo e no CEAPIA entre 1979 e 2007, data do levantamento dos prontuários, foram analisados. Foram excluídos os prontuários referentes à segunda procura por atendimento do mesmo paciente em outro momento e prontuários relativos a pacientes que não assinaram o termo de consentimento, permitindo que os dados relativos aos seus atendimentos fossem usados para fins de ensino e pesquisa.

O banco de dados foi composto e analisado no Statistical Package for the Social Sciences (SPSS), versão 13, com base em um formulário contendo as variáveis sociodemográficas e clínicas extraídas dos prontuários preenchidos pelo responsável pelo paciente, pelo triador do paciente ao chegar às instituições e pelo terapeuta destinado a atender o caso. Os dados transpostos ao banco de dados não incluíam os nomes dos pacientes.

A aderência ao tratamento, desfecho da presente investigação, foi definida com base no tipo de término da psicoterapia descrito nos prontuários. As seguintes definições para término da psicoterapia foram utilizadas ${ }^{3}$ :

- Não aderência: o atendimento é interrompido na fase de avaliação da psicoterapia, ou seja, antes que os objetivos estabelecidos para o tratamento estejam claros para ambos os participantes ou em situações em que não há indicação de tratamento.

- Abandono: a psicoterapia é encerrada antes que os objetivos estabelecidos no contrato tenham sido atingidos, independentemente dos motivos que levaram o paciente ou o terapeuta a interrompê-la e independentemente de a decisão ter sido uni ou bilateral.

- Alta: a psicoterapia é encerrada quando os objetivos estabelecidos no contrato foram atingidos.

Pacientes que abandonaram seus tratamentos e pacientes que tiveram alta foram agrupados e compuseram a categoria aderência, tendo em vista que iniciaram o processo psicoterapêutico e foram além da fase de avaliação.

O motivo de consulta constante nos prontuários não estava padronizado, havendo necessidade de categorização dessa variável para fins de análise. Para proceder a essa categorização, extraíram-se dos prontuários os motivos de consulta informados pelas diferentes partes envolvidas, conforme descrito: 1) pelos pais/responsáveis, ao preencherem a ficha de contato inicial nas instituições; 2) pelo profissional que realizou a triagem daquele paciente, ao preencher a ficha de avaliação na chegada do paciente à instituição; 3) pelo terapeuta responsável pelo caso, ao preencher o roteiro de avaliação psicológica do paciente.

As três primeiras queixas fornecidas em cada um desses formulários foram avaliadas por juízes, os quais categorizaram cada paciente via análise clínica conforme os critérios propostos pelas escalas de comportamento internalizante, 
externalizante, neutra e social do Child Behavior Checklist $(\mathrm{CBCL})^{10}$ - Syndrome Scale 6-18*.

Para a caracterização da amostra, todas as variáveis de interesse foram levantadas e expressas como frequências e porcentagens. Para examinar as relações entre variáveis sociodemográficas e variáveis clínicas e os desfechos aderência/não aderência, foi utilizado o teste do qui-quadrado. Foram considerados significativos os resultados com valor de $\mathrm{p}$ menor do que 0,05 .

\section{Resultados}

Foram analisados prontuários de 2.106 crianças. Destas, 148 ainda estavam em atendimento e 681 apresentavam prontuários incompletos quanto à aderência ou não ao tratamento, sendo possível detectar o desfecho do atendimento de 1.277 pacientes. Após a triagem, constatou-se que 194 pacientes não receberam indicação de psicoterapia, restando 1.083 crianças para a composição da amostra final deste estudo.

A Tabela 1 apresenta as características sociodemográficas da amostra, e a Figura 1 demonstra a distribuição dos pa-

Tabela 1 - Caracterização da amostra quanto às variáveis sociodemográficas

\begin{tabular}{lcc}
\hline Variável & $\mathbf{n}$ & $\mathbf{\%}$ \\
\hline Sexo & & \\
Masculino & 732 & 67,6 \\
Feminino & 351 & 32,4 \\
Idade & & \\
Até 3 anos & 43 & 3,9 \\
4-6 anos & 251 & 23,2 \\
$7-9$ anos & 468 & 43,2 \\
10-12 anos & 321 & 29,6 \\
Escolaridade & & \\
Maternal/creche/berçário & 64 & 6,0 \\
Pré-escola & 125 & 11,5 \\
$1^{\mathrm{a}}$ e 2á séries & 373 & 34,4 \\
$3^{\mathrm{a}}$ série em diante & 324 & 29,9 \\
Não está na escola & 25 & 2,3 \\
Classe especial & 7 & 0,6 \\
Não consta & 165 & 15,2 \\
Configuração familiar & & \\
Ambos os pais & 613 & 56,7 \\
Apenas mãe & 307 & 28,3 \\
Apenas pai & 16 & 1,5 \\
Mãe e padrasto & 66 & 6,1 \\
Pai e madrasta & 9 & 0,8 \\
Outro familiar & 51 & 4,6 \\
Abrigo de proteção & 6 & 0,6 \\
Não consta & 15 & 1,4 \\
Cidade em que reside & & \\
Porto Alegre & 928 & 85,7 \\
Região metropolitana & 131 & 1,1 \\
Interior do estado & 21 & 0,3 \\
Não consta & 3 & \\
Total & 1.083 & \\
\hline & & \\
\hline
\end{tabular}

cientes quanto à época do ano em que buscaram atendimento. Alguns dados não estavam presentes em todos os prontuários, resultando, em algumas variáveis, em uma grande parcela da amostra sem informação disponível.

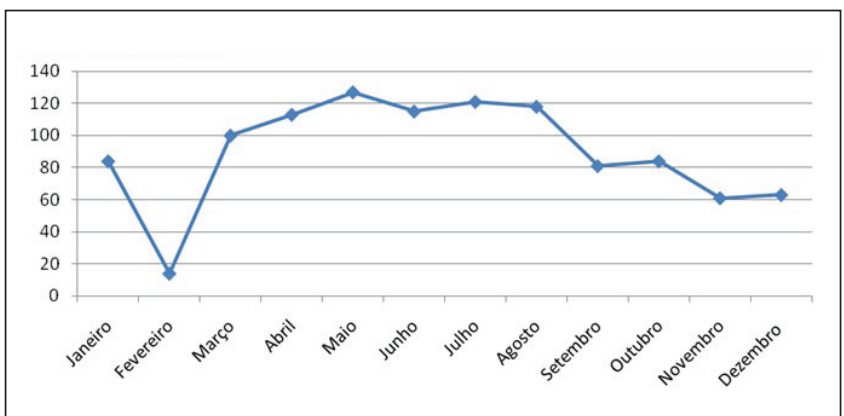

Figura 1 - Distribuição da amostra quanto ao mês do ano em que o paciente buscou atendimento

A Tabela 2 apresenta as variáveis clínicas analisadas, a saber, fonte de encaminhamento, motivo da consulta e uso de medicação.

Tabela 2 - Caracterização da amostra quanto às variáveis clínicas

\begin{tabular}{lcc}
\hline Variável & $\mathbf{n}$ & $\mathbf{\%}$ \\
\hline Fonte de encaminhamento & & \\
Escola & 372 & 34,3 \\
Familiares & 137 & 12,7 \\
Psicólogo & 84 & 7,8 \\
Neurologista & 74 & 6,8 \\
Pediatra & 68 & 6,3 \\
Outra instituição de atendimento & 65 & 6,0 \\
Outras modalidades médicas & 58 & 5,4 \\
Psiquiatra & 36 & 3,3 \\
Conselho Tutelar & 13 & 1,2 \\
Pedagogo & 11 & 1,0 \\
Assistente social & 10 & 0,9 \\
Outros* & 80 & 7,4 \\
Não consta & 75 & 6,9 \\
Motivo de consulta & & \\
Comportamento agressivo & 226 & 20,9 \\
Ansiedade/Depressão & 183 & 16,9 \\
Problemas de atenção & 162 & 15,0 \\
Problemas de aprendizagem & 146 & 13,5 \\
Problemas de relacionamento & 114 & 10,5 \\
Queixas somáticas & 66 & 6,1 \\
Retraimento/Depressão & 65 & 6,0 \\
Comportamento desafiador/opositor & 54 & 5,0 \\
Problemas de pensamento & 32 & 3,0 \\
Não consta & 35 & 3,2 \\
Uso de medicação psiquiátrica ou & & \\
neurológica no momento da triagem & & \\
Não & 969 & 89,5 \\
Sim & 86 & 7,9 \\
Não consta & 28 & 2,6 \\
Total & 1.083 & 100,0 \\
\hline
\end{tabular}

* Foram agrupadas nessa categoria todas as fontes de encaminhamento com menos de 10 crianças, dentre elas: fonoaudiólogo, colega de trabalho dos pais, amigos dos pais, pastores, padres, advogados da família, treinadores desportivos, etc.

* Categorias presentes no CBCL e exemplos de queixas alocadas em cada categoria: Ansiedade/depressão (choros, medos, criança não se sente amada); Retraimento/depressão (tímido, triste, prefere ficar sozinho); Queixas somáticas (tontura, cansaço, náusea, dor de cabeça); Problemas de relacionamento (não se dá bem com as pessoas, dependente, pessoas implicam com a criança); Problemas do pensamento (ouve vozes, vê coisas, comportamentos estranhos); Problemas de atenção (dificuldade de concentração, agitação, devaneios); Comportamento desafiador/opositor (vandalismo, roubos, mentiras); Comportamento agressivo (brigas, gritos, discussões); Problemas de aprendizagem (repetência, dificuldade em alguma disciplina). 
Do total da amostra, 805 pacientes $(78,5 \%)$ demonstraram aderência, comparados com 233 (21,5\%) que não mostraram aderência ao tratamento. Os resultados quanto à relação entre aderência e variáveis sociodemográficas e clínicas são apresentados a seguir.

Aderência e idade. As variáveis idade e aderência não se mostraram associadas nesta amostra (qui-quadrado $=$ $5,239, \mathrm{GL}=3, \mathrm{p}=0,155)$.

Aderência e sexo. Os resultados não mostram associação entre aderência ao tratamento e sexo da criança (qui-quadrado $=1,810, \mathrm{GL}=1, \mathrm{p}=0,179$ ).

Aderência e configuração familiar. A associação entre aderência e configuração familiar foi calculada de duas formas, tendo em vista que a definição de família pode assumir diferentes estruturas de agrupamento ${ }^{11}$. Primeiramente, crianças que moravam com a mãe e o padrasto foram consideradas como morando apenas com a mãe; da mesma forma, as que moravam com o pai e a madrasta foram classificadas como morando apenas com o pai. De acordo com essa opção, não houve associação entre aderência e configuração familiar (qui-quadrado $=6,177, \mathrm{GL}=3, \mathrm{p}=0,103$ ). Um segundo cálculo foi realizado considerando as famílias formadas por padrastos e madrastas como famílias nucleares, nas quais estão presentes as figuras materna e paterna. No entanto, também nessa modalidade, as variáveis aderência e configuração familiar não se mostraram associadas (qui-quadrado $=5,605, \mathrm{GL}=3, \mathrm{p}=0,132$ ). Conforme os resultados obtidos, pode-se concluir que não houve associação entre aderência e configuração familiar nesta amostra, independentemente do tipo de cálculo realizado.

Aderência e escolaridade da criança. As variáveis aderência e escolaridade não se mostraram associadas (qui-quadrado $=4,456, \mathrm{GL}=5, \mathrm{p}=0,486$ ).

Aderência e cidade em que mora. Os resultados indicam não haver relação entre aderência ao tratamento e a cidade de residência da criança, pelo menos para a amostra deste estudo (qui-quadrado $=4,047, \mathrm{GL}=2, \mathrm{p}=0,132$ ).

Aderência e motivo de consulta. Não houve associação entre aderência e motivo de consulta (qui-quadrado = $10,372, \mathrm{GL}=8, \mathrm{p}=0,240$ ).

Aderência e fonte de encaminhamento. Há duas formas possíveis de categorização da fonte de encaminhamento. A primeira opção foi realizar os cálculos mantendo as categorias iniciais. Nesse cálculo, aderência e fonte de encaminhamento mostraram-se associados (qui-quadrado $=30,570$, $\mathrm{GL}=7, \mathrm{p}=0,000$ ), sendo que crianças encaminhadas à psicoterapia pela família aderiram menos que as demais, ao passo que crianças encaminhadas por psiquiatras mostraram-se mais aderentes. A segunda opção foi realizar os cálculos formando novas categorias para fonte de encaminhamento. Para tanto, agruparam-se em uma mesma categoria, denominada "tratamentos combinados", todas as crianças encaminhadas para psicoterapia por pediatras, psiquiatras, neurologistas, demais modalidades médicas, fonoaudiólogos e pedagogos. Entende-se que esses profissionais encaminham crianças à psicoterapia a fim de realizar tratamentos combinados para os problemas do paciente; portanto, o objetivo desse cálculo foi verificar se a realização de tratamentos combinados se associa à aderência à psicoterapia. $\mathrm{O}$ resultado apresentou associação entre as variáveis (qui-quadrado $=20,129, \mathrm{GL}=$ $3, p=0,000$ ), indicando que crianças em tratamentos combinados aderem mais à psicoterapia. Portanto, independentemente do cálculo utilizado, foi observada associação entre aderência à psicoterapia e fonte de encaminhamento para a amostra pesquisada.

Aderência e época do ano em que buscou atendimento. Não houve associação entre aderência ao tratamento e época do ano em que o paciente iniciou atendimento (qui-quadrado $=11,752, \mathrm{GL}=11, \mathrm{p}=0,383)$.

Aderência e uso de medicação neurológica/psiquiátrica no momento da triagem. As variáveis uso de medicação e aderência não se mostraram associadas nesta amostra (qui-quadrado $=2,153, \mathrm{GL}=1, \mathrm{p}=0,142$ ).

$\mathrm{Na}$ amostra pesquisada, portanto, a aderência ao tratamento está associada à fonte de encaminhamento para psicoterapia. A Tabela 3 mostra a distribuição das diferentes fontes de encaminhamento em relação ao desfecho.

Tabela 3 - Distribuição da amostra quanto à aderência de acordo com a fonte de encaminhamento

\begin{tabular}{lcc}
\hline & Não aderência (\%) & Aderência (\%) \\
\hline Familiares & 35,0 & 65,0 \\
Neurologistas & 27,0 & 73,0 \\
Escola & 20,9 & 79,1 \\
Psicólogos & 17,9 & 82,1 \\
Pediatras & 14,7 & 85,3 \\
Outras modalidades médicas & 10,3 & 89,7 \\
Psiquiatras & 2,8 & 97,2 \\
Outros & 22,0 & 78,0 \\
\hline
\end{tabular}

Foi calculado, então, o risco relativo de não aderência, comparando-se as diferentes fontes de encaminhamento. Em comparação com encaminhamentos por psiquiatras, o risco relativo de o paciente não aderir ao tratamento quando encaminhado por outra fonte mostrou-se significativo: 1) no encaminhamento pela família, $R R=1,381$ (IC95\% 1,254$1,520, \mathrm{p}=0,000) ; 2)$ no encaminhamento por neurologistas, $\mathrm{RR}=1,274(\mathrm{IC} 95 \% 1,136-1,429, \mathrm{p}=0,000) ; 3)$ no encaminhamento pela escola, $R R=1,199$ (IC95\% 1,120-1,282, $\mathrm{p}=$ $0,000)$; e 4) no encaminhamento por pediatras, $R R=1,127$ (IC95\% 1,020-1,245, p = 0,019).

Tais resultados indicam que a criança encaminhada pela família apresenta $38 \%$ mais risco de não aderir à psicoterapia do que aquela encaminhada por psiquiatra. As encaminhadas por neurologistas, por sua vez, têm $27 \%$ mais risco de não aderir do que as encaminhadas por psiquiatras. Encaminhamento pela escola aumenta em $20 \%$ o risco de não aderência, e os encaminhamentos por pediatras tem 13\% mais risco de não aderir à psicoterapia quando comparados com encaminhamentos por psiquiatras. Apenas 2,8\% dos encaminhamentos por psiquiatras não aderiram à psicoterapia, 
enquanto $35 \%$ dos encaminhamentos pela família apresentaram esse mesmo desfecho. Assim, o encaminhamento por psiquiatras mostrou-se um fator protetor para a aderência à psicoterapia psicanalítica de crianças.

\section{Discussão}

Esta pesquisa utiliza a mesma amostra de estudo anterior sobre preditores de abandono em psicoterapia psicanalítica de crianças ${ }^{12}$. Por esse motivo, a discussão das características sociodemográficas e clínicas da amostra não será aqui reproduzida, podendo ser encontrada na publicação anterior.

A taxa de não aderência à psicoterapia psicanalítica de crianças neste estudo foi de $21,5 \%$. Ao contrário do que ocorre com o estudo da não aderência ou do abandono na psicoterapia de adultos, há escassez de produção científica sobre o mesmo tema em populações de crianças.

Um dos poucos estudos encontrados ${ }^{13}$, apesar de não ter sido realizado no Brasil, utilizou método de pesquisa semelhante à presente investigação e concluiu que 52,7\% das crianças desistiram do atendimento psicoterápico entre a primeira e a quarta sessão. Ademais, na população geral (adultos e crianças), 50\% dos pacientes, tanto em serviços públicos como em atendimentos privados, desistem do tratamento antes da quinta sessão, segundo dados do National Institute for Mental Health (EUA) ${ }^{14}$.

Comparações e aproximações entre os resultados encontrados por outros autores merecem ser interpretados com cautela no que diz respeito ao desfecho da psicoterapia. Ao revisar a literatura sobre o tema, percebe-se que os autores não coincidem a respeito da definição de não aderência à psicoterapia, utilizando pontos de corte distintos - e, muitas vezes, arbitrários - para distinguir, quando o fazem, não aderência e abandono. Diversos estudos englobam, em um mesmo desfecho, pacientes que não aderiram e que abandonaram seus tratamentos, tamanha a diversidade de definições possíveis para abandono de tratamento encontrada na literatura $^{3}$. Isso dificulta a comparação dos resultados dos diferentes estudos quanto às taxas de prevalência e incidência desse desfecho ${ }^{7,15,16}$.

Em nossa amostra, foi encontrada associação entre fonte de encaminhamento e aderência em psicoterapia psicanalítica de crianças. Crianças encaminhadas à psicoterapia pela família aderiram menos que as demais. Os responsáveis pela criança muitas vezes não estão aptos a identificar com precisão as causas da problemática da criança e, por consequência, não encaminham sua resolução da mesma forma que um profissional especializado o faria. Assim, acabam procurando ajudas inadequadas ou menos efetivas para amenizar os sintomas da criança, frustrando suas expectativas em fases precoces do processo. Ademais, a percepção dos pais acerca dos problemas da criança e a posterior busca de tratamento não é um processo linear e objetivo. Muitas vezes, a motivação parental é ambivalente, sendo que os pais têm a necessidade de lidar com a situação conflitiva de maneira independente ou atenuar a severidade dos sintomas da criança, o que leva à interrupção do tratamento em seu estágio inicial ${ }^{17,18}$. Pesquisa recente ${ }^{19}$ demonstrou a dissintonia de pais e mães na percepção dos problemas da criança, ressaltando a importância de se engajar ambos os integrantes do casal nas fases iniciais do atendimento psicoterapêutico.

Por outro lado, o encaminhamento por médicos psiquiatras foi um fator preventivo à não aderência à psicoterapia psicanalítica de crianças. Possivelmente, a opinião pública associe a imagem dos transtornos mentais com a categoria psiquiátrica, já que essa é a área médica que se dedica especificamente aos problemas de saúde mental ${ }^{20}$. Nos casos em que o encaminhamento foi feito pelo psiquiatra da própria criança, a realização anterior de um atendimento clínico feito pelo psiquiatra pode fornecer informações mais precisas sobre o diagnóstico da criança e esclarecimentos úteis acerca das intervenções terapêuticas, o que fortalece a indicação para psicoterapia e contribui para uma maior aderência ao tratamento psicoterápico ${ }^{21}$.

Outra hipótese seria de que as crianças são encaminhadas à psicoterapia pelos psiquiatras de seus pais. Nesses casos, há um trabalho prévio com o responsável a respeito da necessidade de intervenção para a criança, o que facilita a percepção e aceitação da patologia e o engajamento do responsável na psicoterapia de seu filho.

Também a associação da psicoterapia de crianças com tratamentos combinados psiquiátricos foi um fator preventivo para a não aderência à psicoterapia. Pode-se pensar que a confiança já estabelecida pelos pais anteriormente com algum profissional de saúde pode auxiliar os responsáveis a estabelecer o vínculo terapêutico com maior facilidade na psicoterapia psicanalítica de crianças.

No que concerne ao encaminhamento para psicoterapia, constata-se que, sendo os sintomas externalizantes o principal motivo de consulta em crianças, muitos pacientes infantis acabam sendo encaminhados à psicoterapia porque perturbam o meio em que estão inseridos (a escola, o convívio com amigos, as práticas desportivas, etc.). Na amostra deste estudo, a escola constituiu-se como maior fonte de encaminhamento para psicoterapia, percebendo, antes dos pais, a necessidade de atendimento para as crianças.

Assim, em muitas situações, a fonte de encaminhamento acaba sendo coercitiva, ou seja, os responsáveis não buscaram por conta própria a psicoterapia. Logo, nesses casos, além da avaliação inicial da criança, deve-se ter foco no engajamento do paciente e de seu responsável no processo terapêutico, a fim de tornar a demanda mais interna do que externa, priorizando as intervenções terapêuticas precoces mais nos conflitos intrapsíquicos do que especificamente nas dificuldades ambientais.

Durante a fase inicial de tratamento, além da avaliação clínica da criança, psicoterapeutas psicanalíticos de crianças devem estar atentos para a análise das relações familiares do paciente e, principalmente, para o desenvolvimento de uma 
aliança terapêutica com os pais, com o objetivo de diminuir o risco de interrupção durante as primeiras sessões de atendimento. Com tais evidências científicas, pode-se pensar que o vínculo criado entre o psicoterapeuta e os pais do paciente é, muitas vezes, mais importante para a aderência do tratamento do que a própria relação terapêutica com a criança, validando as implicações de $\operatorname{Kazdin}^{22}$ sobre a psicoterapia nessa faixa etária.

Estudo anterior com a mesma amostra ${ }^{12}$ encontrou os seguintes preditores de abandono de psicoterapia psicanalítica: 1) ser do sexo masculino e 2) ter buscado atendimento encaminhado pela escola ou pela família. Ainda segundo o mesmo estudo, crianças encaminhadas por neurologistas e por psicólogos têm menos risco de abandonar o tratamento do que as demais. A pesquisa atual verificou que o encaminhamento por psiquiatras está associado à aderência à psicoterapia e não encontrou associação entre aderência/não aderência e o sexo da criança. Assim, a principal contribuição da presente investigação é demonstrar que preditores de abandono diferenciam-se de preditores de não aderência à psicoterapia de crianças, mesmo quando comparados dois estudos que utilizaram a mesma amostra e o mesmo método de pesquisa. Tal achado reforça a ideia de que abandono e não aderência à psicoterapia são fenômenos distintos e devem ser interpretados e definidos de forma diferente pelos clínicos.

A associação entre fonte de encaminhamento e aderência à psicoterapia psicanalítica de crianças demonstra que a trajetória realizada anteriormente pelos pais em busca de atendimento para seus filhos é um fenômeno complexo e importante para a fase inicial do tratamento psicoterápico. Diferenças encontradas entre abandono e não aderência nos preditores de fonte de encaminhamento salientam que pesquisas devem ser estimuladas sobre a conduta dos pais na procura de auxílio psicológico para seus filhos, a fim de reduzir barreiras ao acesso a atendimento da saúde mental de crianças $^{23}$.

Sabe-se que não apenas fatores do paciente estão envolvidos na aderência e não aderência à psicoterapia, embora tenha sido este o foco da presente investigação. Keidann \& Dal $Z{ }^{24}{ }^{24}$ abordam que a interrupção em tratamentos psicoterápicos pode estar associada a avaliações iniciais inadequadas e indicações imprecisas realizadas no início do tratamento. Ao receber o paciente, o terapeuta deve fazer uma avaliação inicial e constatar a melhor forma de ajudar o paciente. Tais avaliações preliminares têm o objetivo de verificar indicações ou contraindicações para a demanda. Portanto, a taxa de não aderência à psicoterapia pode estar associada a dificuldades do próprio psicoterapeuta (desconhecimento de técnicas eficazes para determinadas patologias, dificuldades em aceitar as limitações da sua técnica de trabalho, reservas de mercado atuais favorecendo determinados tratamentos, etc.), e não apenas restrita a fatores individuais ou familiares do paciente. Sugere-se, dessa forma, que futuras investigações sejam conduzidas para avaliar os fatores do terapeuta envolvidos no engajamento dos pacientes na psicoterapia de crianças.

Por se tratar de estudo documental, há limitações inerentes ao método da pesquisa. Os resultados apresentados são fruto de análise retrospectiva, a partir de dados coletados de prontuários, apresentando limitações intrínsecas a esse tipo de delineamento. Os dados da pesquisa são derivados de informações constantes nos prontuários dos pacientes, preenchidos subjetivamente por cada terapeuta e dependentes de sua percepção sobre o caso. Ademais, apesar de o estudo ter pesquisado uma grande amostra de crianças em psicoterapia, percebeu-se a falta de informações nos prontuários em diversas variáveis de interesse, limitação própria do estudo documental. Entretanto, tendo em vista a importância clínica desse material, ressalta-se a relevância de pesquisas a partir da prática e da realidade clínica, mesmo considerando suas limitações, em vez da coleta de dados em um ambiente criado deliberadamente para pesquisa. $\mathrm{O}$ interesse crescente em pesquisas naturalísticas reside justamente na tentativa de diminuir a dicotomia entre clínica e pesquisa, uma vez que esse método de investigação tende a aproximar-se mais da prática cotidiana das psicoterapias.

\section{Conclusão}

A presente investigação observou que a fonte de encaminhamento constitui-se como fator preditivo de aderência e não aderência à psicoterapia psicanalítica de crianças. Crianças que são encaminhadas pela família correm mais risco de não aderir à psicoterapia, e as encaminhadas por psiquiatras têm mais facilidade de vinculação ao tratamento durante a fase inicial do processo psicoterápico.

Ademais, demonstrou-se que as variáveis associadas ao abandono da psicoterapia são diferentes das variáveis associadas à não aderência, justificando a necessidade de diferenciação desses dois fenômenos por parte de pesquisadores e clínicos em suas práticas profissionais. Na medida em que poucos estudos usam uma diferenciação conceitual entre abandono e não aderência, os resultados das pesquisas destinadas a avaliar o desfecho da psicoterapia acabam se confundindo, dificultando a generalização dos achados.

Os resultados encontrados são de difícil interpretação, e sua discussão não pretende ser esgotada no presente artigo. A maior contribuição dos achados parece ser lançar luz sobre a questão da não aderência à psicoterapia e fornecer material para que mais teóricos e pesquisadores sigam debruçando-se sobre esse tema, a fim de melhor elucidar o fenômeno. 


\section{Referências}

1. Lancet Global Mental Health Group, Chisholm D, Flisher AJ, Lund C, Patel V, Saxena S, et al. Scale up services for mental disorders: a call for action. Lancet. 2007;307(9594):1247-52.

2. Fleitlich-Bilyk B, Goodman R. Prevalence of child and adolescent psychiatric disorders in southeast Brazil. J Am Acad Child Adolesc Psychiatry. 2004;43:727-34.

3. Gastaud M, Nunes ML. Abandono de tratamento na psicoterapia psicanalítica de crianças [dissertação]. Porto Alegre: Pontifícia Universidade Católica do Rio Grande do Sul; 2008.

4. Urtiaga ME, Almeida G, Vianna MED, Santos MV, Botelho S. Fatores preditivos de abandono em psicoterapias: um estudo na Clínica Sérgio Abuchaim. J Bras Psiquiatr. 1997;6(5):279-83.

5. Lhullier AC. Abandono de tratamento em psicoterapias realizadas numa clínica-escola [tese]. Porto Alegre: Pontifícia Universidade Católica do Rio Grande do Sul; 2002.

6. Campezatto P, Nunes ML. Caracterização da clientela das clínicas-escola de cursos de Psicologia da Região Metropolitana de Porto Alegre. Psicol Reflex Crit. 2007;20(3):376-88.

7. Wierzbicki M, Pekarik G. A meta-analysis of psychotherapy drop-out. Prof Psychol Res Pr. 1993;24:190-5.

8. Miller LM, Southam-Gerow MA, Allin Jr RB. Who stays in treatment? Child and family predictors of youth client retention in a public mental health agency. Child Youth Care Forum. 2008;37:153-70.

9. Estatuto da Criança e do Adolescente. Lei Federal 8.069. Promulgada em 13 de Julho de 1990. Porto Alegre: Conselho Municipal dos Direitos da Criança e do Adolescente; 2000

10. Achenbach TM. Manual for the Child Behavior Checklist/6-18 and 2001 Profile. Burlington: University of Vermont, Department of Psychiatry; 2001.

11. Osório LC. O que é família, afinal? In: Osório LC. Casais e famílias: uma visão contemporânea. Porto Alegre: Artmed; 2002.

12. Gastaud M, Nunes ML. Preditores de abandono de tratamento na psicoterapia psicanalítica de crianças. Rev Psiquiatr Rio Gd Sul. 2009;31(1):13-23.
13. Lazaratou H, Vlassopoulos M, Dellatolas G. Factors affecting compliance with treatment in an outpatient child psychiatric practice: a retrospective study in a community mental health center in Athens. Psychother Psychosom. 2000;69:42-9.

14. Pekarik G. Relationship of expected and actual treatment duration for adult and child clients. J Clin Child Psychol. 1991;20:121-5.

15. Armbruster P, Kazdin AE. Attrition in child psychotherapy. Adv Clin Child Psychol. 1994;16:81-108

16. Hauck S, Kruel L, Sordi A, Sbardellotto G, Cervieri A, Moschetti L, et al. Fatores associados a abandono precoce do tratamento em psicoterapia de orientação analítica. Rev Psiquiatr Rio Gd Sul. 2007;29:265-73.

17. Pavuluri MN, Luk S, McGee R. Help-seeking for behavior problems by parents of preschool children: a community study. J Am Acad Child Adolesc Psychiatry. 1996;35:215-22

18. Douma JCH, Dekker MC, De Ruiter KP, Verhulst FC, Koot HM. Help-seeking process of parents for psychopathology in youth with moderate to 79 borderline intellectual disabilities. J Am Acad Child Adolesc Psychiatry. 2006;45:123242.

19. Borsa JC, Nunes MLT. Concordância parental sobre problemas de comportamento infantil através do CBCL. Paidéia. 2008;18:317-30.

20. Farmer EZ, Burns BJ, Phillips SD, Angold A, Costello EJ. Pathways into and through mental health services for children and adolescents. Psychiatr Serv. 2003;54:60-6.

21. Pescosolido B, Jensen PS, Martin JK, Perry O, Lafsdottir BLS, Fettes D. Public knowledge and assessment of child mental health problems: findings from the National Stigma Study-Children. J Am Acad Child Adolesc Psychiatry. 2008;47:339-49.

22. Kazdin AE. Dropping out of child therapy: issues for research and implications for practice. Clin Child Psychol Psychiatry. 1996;1:133-56.

23. Shanley DC, Reid GJ, Evans B. How parents seek help for children with mental health problems. Adm Policy Ment Health. 2008;35:135-46.

24. Keidann CE, Dal Zot J. Avaliação. In: Eizirik C, Aguiar R, Schestatsky S, eds. Psicoterapia de orientação analítica: fundamentos teóricos e clínicos. Porto Alegre: Artmed; 2005. 